Informal Politics 



\title{
Informal Politics
}

Street Vendors and the State in Mexico City

\author{
John C. Cross
}

Stanford University Press

Stanford, California

1998 
Stanford University Press

Stanford, California

C) 1998 by the Board of Trustees of the

Leland Stanford Junior University

Printed in the United States of America

CIP data appear at the end of the book 